Research Paper

\title{
Short-term effectiveness of inpatient cancer rehabilitation: A longitudinal controlled cohort study
}

\author{
Maria Ture ${ }^{1}$, Felix Angst ${ }^{2}$, André Aeschlimann², Christoph Renner ${ }^{3}$, Ulrich Schnyder ${ }^{4}$, Nic Zerkiebel ${ }^{5}$, Josef \\ Perseus $^{6}$, Jürgen Barth ${ }^{7}$, Marius Bredell ${ }^{1}$, Chantal Martin Soelch ${ }^{8}$, Heinrich Walt ${ }^{1}$, Josef Jenewein ${ }^{\natural}$ \\ 1. Department of Cranio-, Maxillo-Facial and Oral Surgery, University Hospital Zurich, University of Zurich, Zurich, Switzerland \\ 2. RehaClinic Bad Zurzach, Zurzach, Switzerland \\ 3. Oncology Centre Hirslanden Zurich, Switzerland \\ 4. Department of Psychiatry and Psychotherapy, University Hospital Zurich, University of Zurich, Switzerland \\ 5. Susenberg Klinik Zurich, Switzerland \\ 6. Zürcher RehaZentrum Davos, Switzerland \\ 7. Institute of Complementary and Integrative Medicine, University Hospital Zurich, University of Zurich, Switzerland \\ 8. Department of Psychology, Unit of Clinical and Health Psychology, University of Fribourg, Switzerland \\ $\triangle$ Corresponding author: Josef Jenewein, MD, Department of Psychiatry and Psychotherapy, University Hospital Zurich, Rämistrasse 100, 8091 Zurich, \\ Switzerland. Phone: +41 44 255-5257, Fax: +41 44 255-9701, Email address: josef.jenewein@usz.ch \\ (C) Ivyspring International Publisher. This is an open access article distributed under the terms of the Creative Commons Attribution (CC BY-NC) license \\ (https://creativecommons.org/licenses/by-nc/4.0/). See http://ivyspring.com/terms for full terms and conditions.
}

Received: 2017.02.08; Accepted: 2017.05.02; Published: 2017.07.01

\begin{abstract}
Background: Inpatient rehabilitation for cancer patients has been demonstrated to improve patients' health related quality of life (HRQoL) effectively. The purpose of this study was to compare changes in general health and HRQoL of cancer patients who were referred to inpatient rehabilitation (IR) with those in two control groups who underwent outpatient management either with advice for inpatient rehabilitation $\left(A^{+}\right)$or without $\left(A^{-}\right)$.

Methods: In this naturalistic, longitudinal, controlled cohort study, changes in general health and HRQoL were assessed at either discharge of acute hospital or start of rehabilitation (baseline) and at the follow-up 3 weeks later or end of rehabilitation. Outcome variables included general health and HRQoL assessed by the Short Form 36 (SF-36) and the Functional Assessment of Cancer Therapy (FACT), and fatigue (FACT-F), depression and anxiety by the Hospital Anxiety and Depression Scale (HADS). Changes on the scores were compared with bivariate and multivariate analyses using standardized mean differences (SMD).

Results: IR patients $(n=133)$ were on average older, reported lower HRQoL and health, and suffered more frequently from carcinoma than patients of the $A^{+}(n=30)$ and the $A^{-}(n=82)$ groups. In the IR patients, pain, physical functioning, mental health, vitality, and fatigue improved significantly compared to the $\mathrm{A}^{+}$controls. Compared to the $\mathrm{A}$ - group, the bivariate effects were lower but still statistically significant on many scales.

Conclusions: IR showed moderate, statistically significant superior effects over outpatient management of cancer patients after acute treatment. Findings indicate that inpatient cancer rehabilitation can be recommended as an effective management after acute treatment. As today, referrals to inpatient rehabilitation for cancer patients are still not based on structured standardized procedures, the implementation of such screening is needed to address patients' needs and to render the potential for rehabilitation more reliable.
\end{abstract}

Key words: Rehabilitation; Inpatient rehabilitation; Cancer; Effectiveness; Quality of Life; Health; Fatigue; Anxiety; Depression.

\section{Background}

Cancer and cancer therapies, such as surgery, radiotherapy, and chemotherapy, significantly affect physical and psychological health as well as HRQoL [1]. Physical side effects such as functional loss, 
muscle weakness, and fatigue frequently lead to substantial limitations in patients' daily activities [2].

Rehabilitation aims to enable individuals to achieve optimal levels of physical, functional, psychosocial and vocational functioning [3,4]. Further, it helps to maintain social integration and participation in everyday life [5]. Because of the variety of problems cancer patients may face, inpatient rehabilitation programs offer comprehensive multidisciplinary care. Current research shows that rehabilitation improves HRQoL and physical as well as psychological health in cancer patients $[1,6,7]$. In Switzerland, inpatient rehabilitation usually lasts about three weeks [8,9], and consists mainly of physical therapy and other treatments (e.g. nutrition counseling, lymphatic drainage, psychotherapy) [10].

Most studies of cancer rehabilitation have focused on specific interventions, such as exercise programs designed in outpatient settings [11-13]. Some studies have found that inpatient rehabilitation programs had beneficial effects on health and HRQoL [14-18]. However, most of these studies used a pre-post design without control groups. In multidisciplinary rehabilitation programs without control groups, moderate to large effect sizes have been observed in social functioning, vitality, role functioning, and health $[1,19]$. Weis and collaborators found more pronounced improvements in HRQoL for the rehabilitation group than for the control group, partly due to the poorer baseline values of the intervention group [20].

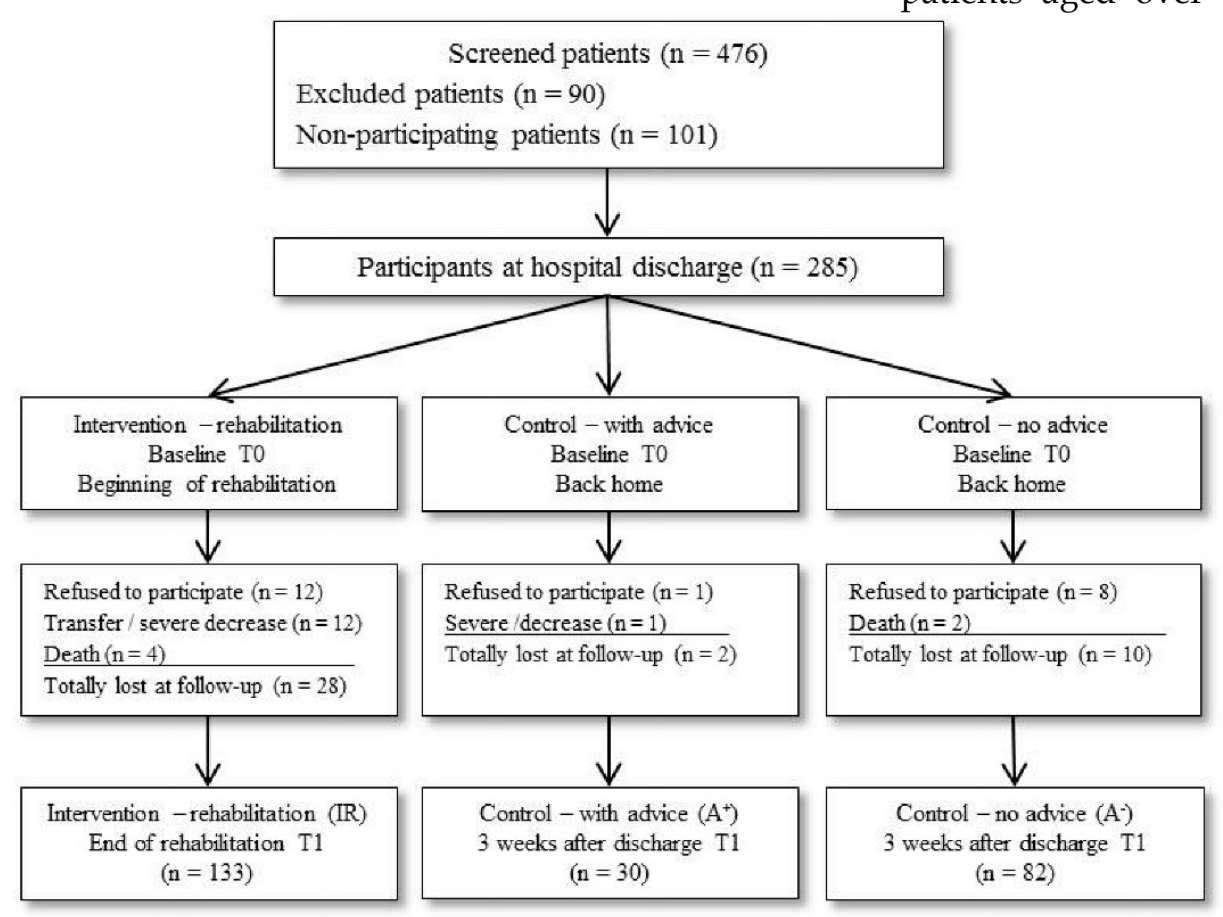

Figure 1. Flow chart of enrolled patients in the study.
Rehabilitation for cancer patients is not well established in Switzerland $[4,14]$. Despite comparable levels of impairments, cancer patients are less likely to use rehabilitation than patients suffering from other chronic diseases [21-24]. Lack of identification of patients' need for rehabilitation, lack of information, and lack of financial support have been identified as major barriers to optimal delivery of rehabilitation care [25].

To our best knowledge, there are currently no empirical data about the effectiveness of inpatient rehabilitation for cancer patients in Switzerland. The main aim of this study was therefore to compare changes in the general health and HRQoL of cancer patients who underwent inpatient rehabilitation (IR) with those in control groups of patients with $\left(\mathrm{A}^{+}\right)$and without $\left(\mathrm{A}^{-}\right)$medical advice for inpatient rehabilitation (outpatient management). We hypothesized that inpatient rehabilitation would lead to greater improvements in general health and HRQoL than outpatient management at home.

\section{Methods}

\section{Participants}

A total of 476 patients who underwent acute treatment for cancer in two hospitals and three rehabilitation clinics in Switzerland between April 2013 and November 2014 were asked to participate in the study. To be eligible, patients had to be 18 years or older. Sufficient German knowledge was required to complete the self-report questionnaires, and all patients aged over 50 years were screened by the German version of the MMSE (Mini Mental State Examination) and excluded if they had a score below 25 points [26] to ensure sufficient cognitive ability to complete the questionnaires. A total of 101 (21\%) patients refused to participate. In addition, 90 (19\%) patients were not included due to severe cognitive or physical impairment or insufficient German language skills (Figure 1).

Of the 285 subjects included, 40 (14\%) dropped out between baseline and follow-up despite reminder calls ( 28 of the IR, 2 of the $\mathrm{A}^{+}$, and 10 of the $\mathrm{A}^{-}$group). Reasons for dropout were 
severe deterioration of health status, re-admission to acute hospital during inpatient rehabilitation (premature end of rehabilitation), death, refusal to participate, and incomplete data. Thirteen $\mathrm{A}^{+}$patients (43\%) did not utilize inpatient rehabilitation because they had to wait too long for rehabilitation, while 17 (57\%) did not receive reimbursement of costs from their health insurance companies. Complete data at baseline (T0) and follow-up (T1) of a total of 245 patients were obtained for analysis: IR $(n=133), A^{+}$ $(\mathrm{n}=30)$, and $\mathrm{A}^{-}(\mathrm{n}=82)$.

\section{Study design}

This multicenter, naturalistic, longitudinal, controlled cohort study grouped participants according to the following types of setting after hospital discharge: a) subjects with referral to rehabilitation who used inpatient rehabilitation (IR); b) subjects with medical advice for inpatient rehabilitation but without reimbursement of costs or subjects who were discharged home from the acute hospital as the waiting time for an available place in the rehabilitation clinic was too long (home with advice, $\left.\mathrm{A}^{+}\right)$; and c) subjects without medical advice for inpatient rehabilitation (home without advice, A-). Written informed consent was obtained from all participants. The study was approved by the Ethical Committee of the Canton of Zurich (KEK-ZH- Nr. 2012-0563).

\section{Procedures}

All participants completed the self-report questionnaires either at discharge from hospital or at the first day of inpatient rehabilitation for those patients who were referred from other hospitals to inpatient rehabilitation (baseline, T0) and three weeks later either at home (control groups) or at the end of rehabilitation (follow-up, T1). Pre- and postoperative treatments and the need for inpatient rehabilitation depended on the case by case decision of the doctors of the acute hospitals. Referrals to inpatient rehabilitation were organized as usual by the social services of hospitals. We did not influence either the referral procedure or the treatment procedure so as to avoid biasing current clinical practice (naturalistic design).

\section{Intervention}

Post-acute hospital inpatient rehabilitation for cancer patients can include comprehensive management. No standardized cancer-specific rehabilitation programs were applied. The use and intensity of treatments were tailored to each individual depending on the severity of impairment, which was assessed at the beginning of rehabilitation. Inpatient rehabilitation includes three to four different treatment components (e.g. physical therapy, nutrition counselling, lymphatic drainage, swallowing therapy, psychotherapy) according to medical prescription. Inpatient rehabilitation can be reimbursed by health insurance companies for two to three weeks. In the $\mathrm{A}^{+}$and $\mathrm{A}^{-}$group, patients received outpatient treatment such as physical therapy, nutrition counselling, and psychotherapy if medically prescribed.

\section{Measures}

Sociodemographic data (gender, age, nationality) and disease-relevant data (insurance type, cancer site, cancer type, treatment, comorbidities) were taken from the patients' clinical records (Table 1). Additional sociodemographic information such as employment status, highest attained education, living status, minor children in household, income, smoking, alcohol consumption and interventions after hospital stay were obtained by standardized questionnaires (Table 1). Tumor stage was classified according to the Tumor-Node-Metastasis (TNM) Classification of Malignant Tumors, except for brain and hematological malignancies [27].

Comprehensive physical, mental, and psycho-social health parameters were assessed using the generic Short-Form (SF-36) Health Survey $[44,28]$. The SF-36 comprises 12 subscales: physical functioning, role physical, bodily pain, general health, vitality, social functioning, role emotional functioning, mental health, physical component summary (PCS), and mental component summary (MCS).

HRQoL was assessed by the disease-specific Functional Assessment of Cancer Therapy General Scale, version 4 (FACT) [29]. The FACT measures the functional, social, emotional, and physical domains of HRQoL (Tables 2 and 3). Fatigue was assessed by the 13-item Fatigue subscale of the FACT measurement system, scaled from $0=$ maximal fatigue to $52=$ no fatigue (FACT-F) [30].

The validated German version of the Hospital Anxiety and Depression Scale (HADS) was used to assess anxiety and depression $[31,32]$. The total score of each scale ranges from 0 (no anxiety/depression) to 21 (maximal anxiety/depression).

\section{Statistics}

Baseline characteristics were compared using the Mann-Whitney U-test for quasi-continuous data and the $\chi^{2}$ test for categorical frequency data. Changes on the scales between baseline and follow-up were firstly expressed by standardized mean differences (bivariate SMD). The SMD represents the difference of the mean score differences (baseline to follow-up) 
between the intervention group (IR) and the control groups $\left(\mathrm{A}^{+}, \mathrm{A}^{-}\right)$divided by the pooled standard deviation of the two groups in each comparison [33]. Intervals for $95 \%$ confidence ( $95 \%-\mathrm{CI})$ were calculated for the SMD and t-test based type I errors (p) for testing SMD > 0.00 (zero outside the 95\%-CI) (Tables 2 and 3). As a rule, an SMD 0.20-0.49 is considered small for effect sizes, $0.50-0.79$ moderate, and $\geq 0.80$ large [34].

Table 1. Patient's sociodemographic and medical characteristics at baseline

\begin{tabular}{|c|c|c|c|c|c|c|}
\hline Characteristics & $\begin{array}{l}\text { Inpatient } \\
\text { rehabilitation (IR) } \\
\mathrm{n}=133(54.3 \%)\end{array}$ & $\begin{array}{l}\text { Control with } \\
\text { Advice } \\
\left(\mathrm{A}^{+}\right) \mathrm{n}=30(\mathbf{1 2 . 2 \% )}\end{array}$ & $\begin{array}{l}\text { Control - } \\
\text { No advice } \\
\left(A^{-}\right) n=82(33.5 \%)\end{array}$ & $\begin{array}{l}\mathrm{p} \\
\text { IR vs. } \mathrm{A}^{+}\end{array}$ & $\begin{array}{l}\mathrm{p} \\
\text { IR vs. } \mathrm{A}^{-}\end{array}$ & $\begin{array}{l}\mathrm{p} \\
\mathrm{A}^{+} \text {vs. } \\
\mathrm{A}^{-}\end{array}$ \\
\hline Age in years (M/IQR) a & $62.0(+/-14.0)$ & $57.5(+/-19.0)$ & $59.0(+/-19.0)$ & 0.020 & 0.012 & 0.610 \\
\hline Hospital stay in days (M/IQR) b & $18.0(+/-9.0)$ & $18.5(+/-12.0)$ & $8.5(+/-8.0)$ & 0.709 & $<0.001$ & $<0.001$ \\
\hline \multicolumn{7}{|l|}{$\operatorname{Sex}(\%)$} \\
\hline Male & $78(59 \%)$ & $19(63 \%)$ & $36(44 \%)$ & 0.637 & 0.035 & 0.069 \\
\hline \multicolumn{7}{|l|}{ Living (\%) } \\
\hline Alone & $42(32 \%)$ & $8(27 \%)$ & $17(21 \%)$ & 0.598 & 0.083 & 0.504 \\
\hline \multicolumn{7}{|l|}{ Children in household (\%) } \\
\hline Yes & $18(14 \%)$ & $6(20 \%)$ & $13(16 \%)$ & 0.367 & 0.638 & 0.605 \\
\hline Level of education (\%) & & & & 0.372 & 0.816 & 0.698 \\
\hline Basic school (8-9 years) & $9(7 \%)$ & $4(13 \%)$ & $8(10 \%)$ & & & \\
\hline Vocational training & $68(51 \%)$ & $15(50 \%)$ & $39(48 \%)$ & & & \\
\hline College/high school & $22(17 \%)$ & $2(7 \%)$ & $12(15 \%)$ & & & \\
\hline University & $34(26 \%)$ & $9(30 \%)$ & $23(28 \%)$ & & & \\
\hline \multicolumn{7}{|l|}{ Employment status } \\
\hline Employed & $46(35 \%)$ & $16(53 \%)$ & $47(57 \%)$ & 0.056 & 0.001 & 0.707 \\
\hline \multicolumn{7}{|l|}{ Insurance type (\%) } \\
\hline Basic & $76(57 \%)$ & $25(83 \%)$ & $63(77 \%)$ & 0.008 & 0.003 & 0.458 \\
\hline Comorbidities (\%) & & & & 0.423 & 0.001 & 0.647 \\
\hline None & $43(32 \%)$ & $14(47 \%)$ & $49(60 \%)$ & & & \\
\hline 1 & $43(32 \%)$ & $8(27 \%)$ & $17(21 \%)$ & & & \\
\hline 2 & $28(21 \%)$ & $6(20 \%)$ & $11(13 \%)$ & & & \\
\hline$>2$ & $19(14 \%)$ & $2(7 \%)$ & $5(6 \%)$ & & & \\
\hline Tumor stage $(\%)$ & & & & 0.994 & 0.001 & 0.065 \\
\hline I & $11(10 \%)$ & $2(9 \%)$ & $17(25 \%)$ & & & \\
\hline II & $27(24 \%)$ & $6(26 \%)$ & $18(27 \%)$ & & & \\
\hline III & $26(23 \%)$ & $5(22 \%)$ & $20(30 \%)$ & & & \\
\hline IV & $51(44 \%)$ & $10(44 \%)$ & $12(18 \%)$ & & & \\
\hline Cancer site (\%) & & & & 0.063 & $<0.001$ & $<0.001$ \\
\hline Digestive organs & $49(37 \%)$ & $6(20 \%)$ & $4(5 \%)$ & & & \\
\hline Thoracic organs & $20(15 \%)$ & $2(7 \%)$ & $5(6 \%)$ & & & \\
\hline Head and neck & $22(17 \%)$ & $13(43 \%)$ & $13(16 \%)$ & & & \\
\hline Hematological malignancies & $15(11 \%)$ & $7(23 \%)$ & $7(9 \%)$ & & & \\
\hline Female genital organs & $7(5 \%)$ & $1(3 \%)$ & $11(13 \%)$ & & & \\
\hline Sarcoma & $5(4 \%)$ & $0(0 \%)$ & $15(18 \%)$ & & & \\
\hline Breast & $8(6 \%)$ & $0(0 \%)$ & $16(20 \%)$ & & & \\
\hline Others & $7(5 \%)$ & $1(3 \%)$ & $11(13 \%)$ & & & \\
\hline Cancer type (\%) & & & & 0.582 & 0.010 & 0.026 \\
\hline Carcinoma & $101(76 \%)$ & $21(70 \%)$ & $50(61 \%)$ & & & \\
\hline Sarcoma & $10(8 \%)$ & $1(3 \%)$ & $15(18 \%)$ & & & \\
\hline Myeloma & $8(6 \%)$ & $3(10 \%)$ & $4(5 \%)$ & & & \\
\hline Lymphoma & $7(5 \%)$ & $4(13 \%)$ & $3(4 \%)$ & & & \\
\hline Mesothelioma & $3(2 \%)$ & $1(3 \%)$ & 0 & & & \\
\hline Brain tumor & $3(2 \%)$ & $0(0 \%)$ & $8(20 \%)$ & & & \\
\hline Others & $1(1 \%)$ & $0(0 \%)$ & $2(2 \%)$ & & & \\
\hline Treatment type $(\%) \mathrm{d}$ & & & & 0.179 & 0008 & 0.597 \\
\hline Surgery & $102(77 \%)$ & $25(83 \%)$ & $74(90 \%)$ & & & \\
\hline Stem cell transplantation & $6(5 \%)$ & $3(10 \%)$ & $5(6 \%)$ & & & \\
\hline Other & $24(18 \%)$ & $2(7 \%)$ & $3(4 \%)$ & & & \\
\hline \multicolumn{7}{|l|}{ Physical therapy } \\
\hline Yes & $130(98 \%)$ & $15(50 \%)$ & $34(42 \%)$ & $<0.001$ & $<0.001$ & 0.420 \\
\hline \multicolumn{7}{|l|}{ Nutrition counselling } \\
\hline Yes & $78(59 \%)$ & $11(37 \%)$ & $10(12 \%)$ & 0.029 & $<0.001$ & 0.003 \\
\hline \multicolumn{7}{|l|}{ Psychological therapy } \\
\hline Yes & $42(32 \%)$ & $2(7 \%)$ & $6(7 \%)$ & 0.005 & $<0.001$ & 1.000 \\
\hline
\end{tabular}

$\mathrm{N}=245$. a M /IQR: Median /interquartile range. ${ }^{b}$ missing ( $n=53$ only IG). ${ }^{c}$ missing $(n=48)$. ${ }^{d}$ missing $(n=1)$. 
Table 2. Comparison of inpatient rehabilitation (IR) with control group with advice $\left(A^{+}\right)$, from T0 to T1

\begin{tabular}{|c|c|c|c|c|c|c|c|c|c|c|c|c|c|}
\hline \multirow[b]{2}{*}{ Scales } & \multicolumn{3}{|c|}{ IR (n=133) } & \multicolumn{2}{|c|}{$\mathrm{A}^{+}(\mathrm{n}=30)$} & \multicolumn{3}{|c|}{ Bivariate } & \multicolumn{5}{|c|}{ Multivariate } \\
\hline & T0 & $\mathrm{T} 1$ & $\Delta$ & T0 & $\mathrm{T} 1$ & $\Delta$ & SMD & $95 \%-\mathrm{CI}$ & $\mathrm{p}$ & SMD & $95 \%-\mathrm{CI}$ & $\mathrm{p}$ & $\mathrm{R}^{2}$ \\
\hline SF-36 Physical functioning & $\begin{array}{l}36.6 \\
(24.2)\end{array}$ & $\begin{array}{l}59.4 \\
(23.6)\end{array}$ & $\begin{array}{l}22.7 \\
(24.2)\end{array}$ & $\begin{array}{l}49.5 \\
(25.1)\end{array}$ & $\begin{array}{l}57.8 \\
(24.8)\end{array}$ & $\begin{array}{l}8.3 \\
(15.8)\end{array}$ & 0.62 & $0.22-1.02$ & 0.001 & 0.62 & $0.22-1.02$ & 0.002 & 37.0 \\
\hline SF-36 Role physical & $\begin{array}{l}22.4 \\
(21.7)\end{array}$ & $\begin{array}{l}43.1 \\
(23.1)\end{array}$ & $\begin{array}{l}20.7 \\
(22.6)\end{array}$ & $\begin{array}{l}29.2 \\
(25.9)\end{array}$ & $\begin{array}{l}39.1 \\
(24.7)\end{array}$ & $\begin{array}{l}9.9 \\
(23.7)\end{array}$ & 0.47 & $0.08-0.87$ & 0.016 & 0.46 & $0.06-0.86$ & 0.023 & 31.1 \\
\hline SF-36 Bodily pain & $\begin{array}{l}48.4 \\
(30.6)\end{array}$ & $\begin{array}{l}65.0 \\
(25.9)\end{array}$ & $\begin{array}{l}16.6 \\
(27.4)\end{array}$ & $\begin{array}{l}56.5 \\
(30.0)\end{array}$ & $\begin{array}{l}60.0 \\
(24.7)\end{array}$ & $\begin{array}{l}3.5 \\
(27.1)\end{array}$ & 0.48 & $0.08-0.87$ & 0.015 & 0.37 & $-0.02-0.77$ & 0.064 & 36.9 \\
\hline SF-36 General health & $\begin{array}{l}51.0 \\
(19.1)\end{array}$ & $\begin{array}{l}56.6 \\
(18.8)\end{array}$ & $\begin{array}{l}5.6 \\
(16.3)\end{array}$ & $\begin{array}{l}56.2 \\
(21.8)\end{array}$ & $\begin{array}{l}53.9 \\
(16.1)\end{array}$ & $\begin{array}{l}-2.2 \\
(14.3)\end{array}$ & 0.49 & $0.09-0.89$ & 0.012 & 0.40 & $0.00-0.80$ & 0.047 & 27.9 \\
\hline SF-36 Vitality & $\begin{array}{l}36.9 \\
(21.3)\end{array}$ & $\begin{array}{l}52.4 \\
(21.7)\end{array}$ & $\begin{array}{l}15.5 \\
(20.7)\end{array}$ & $\begin{array}{l}42.0 \\
(25.5)\end{array}$ & $\begin{array}{l}45.1 \\
(20.9)\end{array}$ & $\begin{array}{l}3.0 \\
(13.2)\end{array}$ & 0.64 & $0.23-1.04$ & 0.001 & 0.64 & $0.24-1.04$ & 0.002 & 39.9 \\
\hline SF-36 Social functioning & $\begin{array}{l}58.0 \\
(33.5)\end{array}$ & $\begin{array}{l}73.6 \\
(28.6)\end{array}$ & $\begin{array}{l}15.6 \\
(36.2)\end{array}$ & $\begin{array}{l}61.0 \\
(34.7)\end{array}$ & $\begin{array}{l}57.7 \\
(28.3)\end{array}$ & $\begin{array}{l}-3.3 \\
(30.6)\end{array}$ & 0.53 & $0.14-0.93$ & 0.006 & 0.70 & $0.30-1.10$ & 0.001 & 52.5 \\
\hline SF-36 Role emotional & $\begin{array}{l}50.9 \\
(33.6)\end{array}$ & $\begin{array}{l}64.5 \\
(29.5)\end{array}$ & $\begin{array}{l}13.6 \\
(34.4)\end{array}$ & $\begin{array}{l}55.6 \\
(33.4)\end{array}$ & $\begin{array}{l}62.6 \\
(31.4)\end{array}$ & $\begin{array}{l}7.0 \\
(31.4)\end{array}$ & 0.19 & $-0.20-0.59$ & 0.332 & 0.21 & $-0.18-0.61$ & 0.296 & 44.0 \\
\hline SF-36 Mental Health & $\begin{array}{l}65.5 \\
(19.7)\end{array}$ & $\begin{array}{l}73.5 \\
(18.3)\end{array}$ & $\begin{array}{l}8.0 \\
(18.2)\end{array}$ & $\begin{array}{l}63.7 \\
(20.5)\end{array}$ & $\begin{array}{l}65.3 \\
(17.5)\end{array}$ & $\begin{array}{l}1.7 \\
(14.8)\end{array}$ & 0.36 & $-0.04-0.75$ & 0.070 & 0.46 & $0.06-0.86$ & 0.023 & 31.7 \\
\hline SF-36 PCS & $\begin{array}{l}32.2 \\
(8.0)\end{array}$ & $\begin{array}{l}40.0 \\
(8.0)\end{array}$ & $\begin{array}{l}7.8 \\
(7.9)\end{array}$ & $\begin{array}{l}37.0 \\
(9.3)\end{array}$ & $\begin{array}{l}39.3 \\
(7.5)\end{array}$ & $\begin{array}{l}2.3 \\
(7.0)\end{array}$ & 0.71 & $0.31-1.11$ & $<0.001$ & 0.59 & $0.20-0.99$ & 0.003 & 34.2 \\
\hline SF-36 MCS & $\begin{array}{l}42.9 \\
(12.6)\end{array}$ & $\begin{array}{l}47.6 \\
(11.9)\end{array}$ & $\begin{array}{l}4.7 \\
(12.3)\end{array}$ & $\begin{array}{l}42.4 \\
(13.2)\end{array}$ & $\begin{array}{l}42.8 \\
(11.8)\end{array}$ & $\begin{array}{l}0.4 \\
(10.0)\end{array}$ & 0.35 & $-0.04-0.75$ & 0.073 & 0.46 & $0.06-0.86$ & 0.025 & 38.7 \\
\hline FACT Physical & $\begin{array}{l}15.9 \\
(6.1)\end{array}$ & $\begin{array}{l}20.3 \\
(5.1)\end{array}$ & $\begin{array}{l}4.5 \\
(5.5)\end{array}$ & $\begin{array}{l}17.6 \\
(6.4)\end{array}$ & $\begin{array}{l}18.8 \\
(5.6)\end{array}$ & $\begin{array}{l}1.2 \\
(4.7)\end{array}$ & 0.61 & $0.21-1.01$ & 0.002 & 0.51 & $0.11-0.91$ & 0.012 & 40.6 \\
\hline FACT Social & $\begin{array}{l}24.3 \\
(3.8)\end{array}$ & $\begin{array}{l}24.1 \\
(3.7)\end{array}$ & $\begin{array}{l}-0.1 \\
(2.8)\end{array}$ & $\begin{array}{l}22.1 \\
(5.6)\end{array}$ & $\begin{array}{l}22.2 \\
(5.9)\end{array}$ & $\begin{array}{l}0.1 \\
(2.9)\end{array}$ & -0.08 & $-0.47-0.32$ & 0.695 & 0.01 & $-0.38-0.41$ & 0.953 & 18.5 \\
\hline FACT Emotional & $\begin{array}{l}18.7 \\
(4.3)\end{array}$ & $\begin{array}{l}19.7 \\
(4.0)\end{array}$ & $\begin{array}{l}0.9 \\
(3.2)\end{array}$ & $\begin{array}{l}18.6 \\
(4.1)\end{array}$ & $\begin{array}{l}18.8 \\
(3.6)\end{array}$ & $\begin{array}{l}0.2 \\
(3.4)\end{array}$ & 0.22 & $-0.17-0.62$ & 0.270 & 0.19 & $-0.20-0.58$ & 0.346 & 25.2 \\
\hline FACT Functional & $\begin{array}{l}12.9 \\
(6.3)\end{array}$ & $\begin{array}{l}16.4 \\
(6.1)\end{array}$ & $\begin{array}{l}3.6 \\
(6.0)\end{array}$ & $\begin{array}{l}13.4 \\
(5.9)\end{array}$ & $\begin{array}{l}14.5 \\
(5.9)\end{array}$ & $\begin{array}{l}1.1 \\
(4.9)\end{array}$ & 0.42 & $0.03-0.82$ & 0.032 & 0.42 & $0.02-0.82$ & 0.039 & 24.2 \\
\hline FACT Total & $\begin{array}{l}71.7 \\
(15.0)\end{array}$ & $\begin{array}{l}80.6 \\
(15.1)\end{array}$ & $\begin{array}{l}8.8 \\
(13.1)\end{array}$ & $\begin{array}{l}71.8 \\
(15.4)\end{array}$ & $\begin{array}{l}74.3 \\
(14.4)\end{array}$ & $\begin{array}{l}2.6 \\
(11.3)\end{array}$ & 0.49 & $0.09-0.88$ & 0.013 & 0.48 & $0.09-0.88$ & 0.017 & 23.2 \\
\hline FACIT Fatigue & $\begin{array}{l}25.1 \\
(12.1)\end{array}$ & $\begin{array}{l}34.3 \\
(11.5)\end{array}$ & $\begin{array}{l}9.2 \\
(11.3)\end{array}$ & $\begin{array}{l}29.2 \\
(13.6)\end{array}$ & $\begin{array}{l}31.6 \\
(11.6)\end{array}$ & $\begin{array}{l}2.4 \\
(10.4)\end{array}$ & 0.61 & $0.21-1.01$ & 0.002 & 0.54 & $0.14-0.94$ & 0.008 & 37.3 \\
\hline HADS Anxiety & $\begin{array}{l}4.8 \\
(3.2)\end{array}$ & $\begin{array}{l}4.0 \\
(3.3)\end{array}$ & $\begin{array}{l}-0.8 \\
(3.2)\end{array}$ & $\begin{array}{l}4.9 \\
(2.6)\end{array}$ & $\begin{array}{l}5.0 \\
(3.0)\end{array}$ & $\begin{array}{l}0.1 \\
(2.8)\end{array}$ & 0.28 & $-0.11-0.68$ & 0.154 & 0.37 & $-0.03-0.76$ & 0.070 & 24.3 \\
\hline HADS Depression & $\begin{array}{l}6.8 \\
(4.6)\end{array}$ & $\begin{array}{l}5.2 \\
(4.5)\end{array}$ & $\begin{array}{l}-1.6 \\
(3.6)\end{array}$ & $\begin{array}{l}6.0 \\
(3.8)\end{array}$ & $\begin{array}{l}6.6 \\
(4.2)\end{array}$ & $\begin{array}{l}0.6 \\
(3.2)\end{array}$ & 0.62 & $0.22-1.02$ & 0.001 & 0.53 & $0.13-0.93$ & 0.009 & 20.9 \\
\hline
\end{tabular}

Table 3. Comparison of inpatient rehabilitation (IR) with control group - no advice (A-), from T0 to T1

\begin{tabular}{|c|c|c|c|c|c|c|c|c|c|c|c|c|c|}
\hline \multirow[b]{2}{*}{ Scales } & \multicolumn{3}{|c|}{ IR $(n=133)$} & \multicolumn{2}{|c|}{$A^{-}(n=82)$} & \multicolumn{3}{|c|}{ Bivariate } & \multicolumn{5}{|c|}{ Multivariate } \\
\hline & T0 & $\mathrm{T} 1$ & $\Delta$ & T0 & $\mathrm{T} 1$ & $\Delta$ & SMD & $95 \%-\mathrm{CI}$ & $\mathrm{p}$ & SMD & $95 \%-C I$ & $\mathrm{p}$ & $\mathrm{R}^{2}$ \\
\hline SF-36 Physical functioning & $\begin{array}{l}36.6 \\
(24.2)\end{array}$ & $\begin{array}{l}59.4 \\
(23.6)\end{array}$ & $\begin{array}{l}22.7 \\
(24.2)\end{array}$ & $\begin{array}{l}51.0 \\
(25.4)\end{array}$ & $\begin{array}{l}67.2 \\
(20.4)\end{array}$ & $\begin{array}{l}16.2 \\
(21.2)\end{array}$ & 0.28 & $0.01-0.56$ & 0.040 & -0.06 & $-0.33-0.22$ & 0.674 & 37.0 \\
\hline SF-36 Role physical & $\begin{array}{l}22.4 \\
(21.7)\end{array}$ & $\begin{array}{l}43.1 \\
(23.1)\end{array}$ & $\begin{array}{l}20.7 \\
(22.6)\end{array}$ & $\begin{array}{l}34.8 \\
(24.0)\end{array}$ & $\begin{array}{l}45.1 \\
(22.4)\end{array}$ & $\begin{array}{l}10.3 \\
(22.0)\end{array}$ & 0.46 & $0.19-0.74$ & 0.001 & 0.20 & $-0.08-0.47$ & 0.160 & 31.1 \\
\hline SF-36 Bodily pain & $\begin{array}{l}48.4 \\
(30.6)\end{array}$ & $\begin{array}{l}65.0 \\
(25.9)\end{array}$ & $\begin{array}{l}16.6 \\
(27.4)\end{array}$ & $\begin{array}{l}50.9 \\
(26.8)\end{array}$ & $\begin{array}{l}64.8 \\
(26.9)\end{array}$ & $\begin{array}{l}13.9 \\
(25.9)\end{array}$ & 0.10 & $-0.17-0.37$ & 0.475 & 0.06 & $-0.22-0.33$ & 0.683 & 36.9 \\
\hline SF-36 General health & $\begin{array}{l}51.0 \\
(19.1)\end{array}$ & $\begin{array}{l}56.6 \\
(18.8)\end{array}$ & $\begin{array}{l}5.6 \\
(16.3)\end{array}$ & $\begin{array}{l}62.7 \\
(17.8)\end{array}$ & $\begin{array}{l}62.0 \\
(18.7)\end{array}$ & $\begin{array}{l}-0.7 \\
(15.2)\end{array}$ & 0.40 & $0.12-0.67$ & 0.003 & 0.07 & $-0.21-0.34$ & 0.633 & 27.9 \\
\hline SF-36 Vitality & $\begin{array}{l}36.9 \\
(21.3)\end{array}$ & $\begin{array}{l}52.4 \\
(21.7)\end{array}$ & $\begin{array}{l}15.5 \\
(20.7)\end{array}$ & $\begin{array}{l}49.4 \\
(23.9)\end{array}$ & $\begin{array}{l}54.2 \\
(19.3)\end{array}$ & $\begin{array}{l}4.7 \\
(19.0)\end{array}$ & 0.54 & $0.26-0.81$ & $<0.001$ & 0.28 & $0.00-0.55$ & 0.047 & 39.3 \\
\hline SF-36 Social functioning & $\begin{array}{l}58.0 \\
(33.5)\end{array}$ & $\begin{array}{l}73.6 \\
(28.6)\end{array}$ & $\begin{array}{l}15.6 \\
(36.2)\end{array}$ & $\begin{array}{l}67.4 \\
(31.9)\end{array}$ & $\begin{array}{l}71.5 \\
(25.0)\end{array}$ & $\begin{array}{l}4.1 \\
(30.6)\end{array}$ & 0.33 & $0.06-0.61$ & 0.014 & 0.10 & $-0.17-0.38$ & 0.458 & 52.5 \\
\hline SF-36 Role emotional & $\begin{array}{l}50.9 \\
(33.6)\end{array}$ & $\begin{array}{l}64.5 \\
(29.5)\end{array}$ & $\begin{array}{l}13.6 \\
(34.4)\end{array}$ & $\begin{array}{l}64.5 \\
(30.6)\end{array}$ & $\begin{array}{l}67.9 \\
(28.3)\end{array}$ & $\begin{array}{l}3.4 \\
(36.4)\end{array}$ & 0.29 & $0.01-0.57$ & 0.034 & 0.03 & $-0.24-0.31$ & 0.824 & 44.0 \\
\hline SF-36 Mental Health & $\begin{array}{l}65.5 \\
(19.7)\end{array}$ & $\begin{array}{l}73.5 \\
(18.3)\end{array}$ & $\begin{array}{l}8.0 \\
(18.2)\end{array}$ & $\begin{array}{l}68.7 \\
(19.6)\end{array}$ & $\begin{array}{l}72.0 \\
(20.1)\end{array}$ & $\begin{array}{l}3.3 \\
(15.6)\end{array}$ & 0.27 & $0.00-0.55$ & 0.046 & 0.07 & $-0.20-0.35$ & 0.601 & 31.7 \\
\hline SF-36 PCS & $\begin{array}{l}32.2 \\
(8.0)\end{array}$ & $\begin{array}{l}40.0 \\
(8.0)\end{array}$ & $\begin{array}{l}7.8 \\
(7.9)\end{array}$ & $\begin{array}{l}36.7 \\
(8.4)\end{array}$ & $\begin{array}{l}42.2 \\
(7.8)\end{array}$ & $\begin{array}{l}5.4 \\
(7.0)\end{array}$ & 0.31 & $0.03-0.58$ & 0.025 & 0.05 & $-0.23-0.32$ & 0.734 & 34.2 \\
\hline SF-36 MCS & $\begin{array}{l}42.9 \\
(12.6)\end{array}$ & $\begin{array}{l}47.6 \\
(11.9)\end{array}$ & $\begin{array}{l}4.7 \\
(12.3)\end{array}$ & $\begin{array}{l}46.9 \\
(12.1)\end{array}$ & $\begin{array}{l}46.9 \\
(12.4)\end{array}$ & $\begin{array}{l}0.0 \\
(12.1)\end{array}$ & 0.38 & $0.10-0.66$ & 0.005 & 0.16 & $-0.11-0.44$ & 0.241 & 38.7 \\
\hline FACT Physical & $\begin{array}{l}15.9 \\
(6.1)\end{array}$ & $\begin{array}{l}20.3 \\
(5.1)\end{array}$ & $\begin{array}{l}4.5 \\
(5.5)\end{array}$ & $\begin{array}{l}18.6 \\
(5.5)\end{array}$ & $\begin{array}{l}20.8 \\
(5.1)\end{array}$ & $\begin{array}{l}2.1 \\
(5.4)\end{array}$ & 0.43 & $0.15-0.70$ & 0.002 & 0.13 & $-0.14-0.41$ & 0.346 & 40.6 \\
\hline FACT Social & $\begin{array}{l}24.3 \\
(3.8)\end{array}$ & $\begin{array}{l}24.1 \\
(3.7)\end{array}$ & $\begin{array}{l}-0.1 \\
(2.8)\end{array}$ & $\begin{array}{l}24.2 \\
(3.5)\end{array}$ & $\begin{array}{l}24.0 \\
(3.5)\end{array}$ & $\begin{array}{l}-0.2 \\
(2.9)\end{array}$ & 0.02 & $-0.25-0.30$ & 0.876 & 0.02 & $-0.25-0.30$ & 0.880 & 18.5 \\
\hline
\end{tabular}




\begin{tabular}{|c|c|c|c|c|c|c|c|c|c|c|c|c|c|}
\hline FACT Emotional & $\begin{array}{l}18.7 \\
(4.3)\end{array}$ & $\begin{array}{l}19.7 \\
(4.0)\end{array}$ & $\begin{array}{l}0.9 \\
(3.2)\end{array}$ & $\begin{array}{l}19.7 \\
(3.8)\end{array}$ & $\begin{array}{l}19.8 \\
(3.6)\end{array}$ & $\begin{array}{l}0.1 \\
(2.7)\end{array}$ & 0.26 & $-0.02-0.54$ & 0.058 & 0.09 & $-0.19-0.36$ & 0.536 & 25.2 \\
\hline FACT Functional & $\begin{array}{l}12.9 \\
(6.3)\end{array}$ & $\begin{array}{l}16.4 \\
(6.1)\end{array}$ & $\begin{array}{l}3.6 \\
(6.0)\end{array}$ & $\begin{array}{l}16.0 \\
(5.7)\end{array}$ & $\begin{array}{l}17.7 \\
(6.1)\end{array}$ & $\begin{array}{l}1.8 \\
(4.1)\end{array}$ & 0.33 & $0.06-0.61$ & 0.015 & 0.11 & $-0.16-0.39$ & 0.420 & 24.2 \\
\hline FACT Total & $\begin{array}{l}71.7 \\
(15.0)\end{array}$ & $\begin{array}{l}80.6 \\
(15.1)\end{array}$ & $\begin{array}{l}8.8 \\
(13.1)\end{array}$ & $\begin{array}{l}78.4 \\
(14.5)\end{array}$ & $\begin{array}{l}82.3 \\
(15.1)\end{array}$ & $\begin{array}{l}3.9 \\
(10.8)\end{array}$ & 0.40 & $0.13-0.68$ & 0.003 & 0.20 & $-0.07-0.48$ & 0.150 & 23.2 \\
\hline FACIT Fatigue & $\begin{array}{l}25.1 \\
(12.1)\end{array}$ & $\begin{array}{l}34.3 \\
(11.5)\end{array}$ & $\begin{array}{l}9.2 \\
(11.3)\end{array}$ & $\begin{array}{l}33.2 \\
(11.4)\end{array}$ & $\begin{array}{l}35.5 \\
(10.1)\end{array}$ & $\begin{array}{l}2.2 \\
(8.9)\end{array}$ & 0.66 & $0.38-0.94$ & $<0.001$ & 0.29 & $0.01-0.56$ & 0.042 & 37.3 \\
\hline HADS Anxiety & $\begin{array}{l}4.8 \\
(3.2)\end{array}$ & $\begin{array}{l}4.0 \\
(3.3)\end{array}$ & $\begin{array}{l}-0.8 \\
(3.2)\end{array}$ & $\begin{array}{l}4.9 \\
(3.6)\end{array}$ & $\begin{array}{l}4.5 \\
(3.5)\end{array}$ & $\begin{array}{l}-0.4 \\
(2.3)\end{array}$ & 0.14 & $-0.13-0.42$ & 0.308 & 0.07 & $-0.20-0.35$ & 0.601 & 24.3 \\
\hline HADS Depression & $\begin{array}{l}6.8 \\
(4.6)\end{array}$ & $\begin{array}{l}5.2 \\
(4.5)\end{array}$ & $\begin{array}{l}-1.6 \\
(3.6)\end{array}$ & $\begin{array}{l}4.9 \\
(3.6)\end{array}$ & $\begin{array}{l}4.6 \\
(4.1)\end{array}$ & $\begin{array}{l}-0.3 \\
(2.9)\end{array}$ & 0.37 & $0.10-0.65$ & 0.006 & 0.15 & $-0.13-0.42$ & 0.294 & 20.9 \\
\hline
\end{tabular}

Legend: All values are arithmetic mean, standard deviation in parentheses. SF-36= Short Form Health Short-Form Health Survey Index ranging from $0=$ low health to $100=$ maximal health, FACT-G $=$ Functional Assessment of Cancer Therapy from $0=$ low HRQoL to $108=$ maximal HRQoL, FACIT-F $=$ Functional Assessment Cancer Therapy Fatigue from $0=$ maximal Fatigue to $52=$ low Fatigue, HADS $=$ Hospital Anxiety Depression Scale from $0=$ no anxiety $/$ depression to $21=$ maximal anxiety $/$ depression, $\mathrm{n}$ $=$ number of complete data sets, $\mathrm{T} 0=$ hospital discharge $/$ beginning of rehabilitation, $\Delta=$ difference $(\mathrm{T} 1-\mathrm{T} 0)$, $\mathrm{R}^{2}=$ Explained variance

For each score, multivariate SMDs were then obtained using the individual score differences (baseline to follow-up) as dependent variable for stepwise multivariate linear regression modeling. The multivariate SMDs were controlled for differences between the intervention and control groups to identify potential confounders (independent variables), namely the different baseline scores of the scales under examination, gender, age, number of comorbidities, and cancer site.

The corresponding dependent variable at baseline, the grouping variable for $\mathrm{A}^{+}\left(1\right.$ for $\mathrm{A}^{+}, 0$ otherwise), and the grouping variable for $\mathrm{A}^{-}\left(1\right.$ for $\mathrm{A}^{-}$, 0 otherwise), were included in the multivariate analysis. By so doing, the regression coefficients of the grouping variables equaled the differences between modelled mean score differences (baseline to follow-up) between intervention and control. These raw, mean score differences were then used to compute SMDs [33]. The pooled standard deviations for the SMDs were determined by the standard errors of the raw differences obtained by the regression models. All statistical analyses were performed using the Statistical Package for the Social Sciences (SPSS) version 21.0 for Windows (SPSS Inc., Chicago, IL, USA).

\section{Results}

\section{Participants}

Sociodemographic data and disease-relevant characteristics are presented in Table 1. Compared to both control groups at T0, the IR patients were more likely to be older and less often employed, and had semi-private/private insurance more often. The IR and the $\mathrm{A}^{+}$stayed longer in hospital, had higher tumor stage, and suffered more frequently from carcinoma. In addition, the IR had more comorbidities and received more interventions during follow-up. Patients who underwent surgery were more likely to be discharged home. Patients with cancer of digestive organs or head and neck were more likely to be referred to inpatient rehabilitation, whereas patients with breast cancer or cancer of female genital organs were less likely to be referred. There were no significant differences between the three groups regarding living status, minor children in household, education level, income, smoking, or risk drinking.

\section{Effects of inpatient rehabilitation compared to subjects with advice for inpatient rehabilitation}

Compared to the $\mathrm{A}^{+}(\mathrm{n}=30)$, the IR patients $(\mathrm{n}=$ 133) showed significant improvements with regard to SF-36 bodily pain (SMD $=0.48$ (bivariate) $/ 0.37$ (multivariate)), SF-36 physical functioning $(\mathrm{SMD}=0.62 / 0.62), \quad \mathrm{SF}-36$ physical component summary $\quad(\mathrm{SMD}=0.71 / 0.59), \quad \mathrm{SF}-36 \quad$ vitality $(\mathrm{SMD}=0.64 / 0.64)$, and SF-36 mental health $(\mathrm{SMD}=0.36 / 0.46)$. The FACT test showed significant improvements in the physical $(\mathrm{SMD}=0.61 / 0.51)$ and functional $(\mathrm{SMD}=0.42 / 0.42)$ dimensions, the FACT total score $(\mathrm{SMD}=0.49 / 0.48)$, and fatigue $(\mathrm{SMD}=0.61 / 0.54)$. IR patients also showed a significant improvement regarding HADS depression $(\mathrm{SMD}=0.62 / 0.43)$ (Table 2). In total, of the 18 health dimensions, 7 bivariate SMDs and 4 multivariate SMDs were $\geq 0.50$, and 12 bivariate and 11 multivariate effects were statistically significant at an alpha level $p<0.05$. No significant multivariate effects were found in emotional role (SF-36) or in FACT social and emotional dimensions.

At baseline, SF-36 physical functioning (means: 36.6 versus $49.5, \mathrm{p}=0.008$ ) and SF-36 PCS (means: 32.2 versus $37.0, p=0.014$ ) were significantly poorer in the IR group than in the $\mathrm{A}^{+}$group. In addition, social support was significantly higher in the IR (means 24.3 versus 22.1, $\mathrm{p}=0.013$ ). However, controlling for these baseline differences by multivariate regression modeling did not reduce the multivariate SMDs by large amounts when compared to the bivariate, for instance, SMDs of SF-36 physical functioning: 0.62 versus 0.62 . The overall fit of the models in predicting the effect differences with the confounders ranged 
from $18.5 \%$ (FACT social) to $52.5 \%$ (SF-36 social functioning).

\section{Effects of inpatient rehabilitation compared to subjects without advice for inpatient rehabilitation}

IR patients $(n=133)$ had higher improvements than the $\mathrm{A}^{-}(\mathrm{n}=82)$ regarding SF-36 vitality $(\mathrm{SMD}=0.54 \quad$ (bivariate) $/ 0.28$ (multivariate)), SF-36 physical functioning $(\mathrm{SMD}=0.31 / 0.05), \mathrm{SF}-36$ mental health $(\mathrm{SMD}=0.38 / 0.16), \quad \mathrm{FACT}$ physical $(\mathrm{SMD}=0.43 / 0.13)$, FACT functional $(\mathrm{SMD}=0.33 / 0.11)$, FACT total $(\mathrm{SMD}=0.40 / 0.20)$, FACT fatigue $(\mathrm{SMD}=0.66 / 0.29)$, and HADS depression $(\mathrm{SMD}=0.37 / 0.15) \quad$ (Table 3). Of the 18 health dimensions, 2 bivariate SMDs were $\geq 0.50,14$ bivariate and 1 multivariate effects were statistically significant at type I error level $\mathrm{p}<0.050$.

At baseline, function (all scales of SF-36 except bodily pain and FACT), mental health (SF-36, HADS depression), and fatigue were significantly poorer in the IR group than in the $\mathrm{A}^{-}$group $(\mathrm{p}<0.001$ to $\mathrm{p}=0.044$ ). For example, SF-36 physical functioning (means: 36.6 versus 51.0, $\mathrm{p}<0.001$ ) and SF-36 PCS (means: 32.2 versus $36.7, \mathrm{p}<0.001$ ) were significantly poorer in the IR group than in the $\mathrm{A}^{-}$group. Controlling for these baseline differences by multivariate regression modeling did not reduce the multivariate SMDs by large amounts when compared to the bivariate, for instance, SMDs of SF-36 vitality: 0.54 versus 0.28 . The overall fit of the models in predicting the effect differences with the confounders ranged from $18.5 \%$ (FACT social) to $52.5 \%$ (SF-36 social functioning). The most important confounder was the baseline score of the dependent variables $(p<0.001$ for all scales). Age was a significant confounder for the SF-36 physical functioning $(p=0.023)$. Female genital organ cancer was a confounder for the SF-36 social functioning $(p=0.024)$ and SF-36 MCS $(\mathrm{p}=0.007)$.

\section{Discussion}

To our best knowledge, this is the first study using a naturalistic, longitudinal, comparative cohort design on the effectiveness of inpatient cancer rehabilitation including control groups in Switzerland. Moderate, statistically significant effect sizes reflecting improvements in favor of inpatient rehabilitation were observed in important health dimensions.

Over the observation period, patients referred to inpatient rehabilitation showed significant improvements in physical and psychosocial health, HRQoL, fatigue, anxiety, and depression, attaining small to moderate size effects. In particular, the effects of the IR to $\mathrm{A}^{+}$comparison were higher and less affected by baseline differences in the scales than those of the IR to $\mathrm{A}^{-}$comparison. In contrast, compared to those patients who did not receive advice for rehabilitation, the effects were considerably smaller and nearly disappeared when controlled for confounders (multivariate SMDs close to zero).

Our findings are consistent with previous research showing an improvement of health and HRQoL after inpatient rehabilitation [14-16,35]. However, most previous studies compared inpatient rehabilitation with a specific additional intervention or design [36,37]. Similarly to our study, Weis et al. had a control group of patients who did not use inpatient rehabilitation [20]. In contrast to their study, our main control group $\left(\mathrm{A}^{+}\right)$and intervention group (IR) had comparable scores at baseline. They found improvements in both groups but higher effect sizes in the rehabilitation group. The largest effects were observed in fatigue for both groups, with an effect size of $d=0.50$. Both of Weis et al.'s groups differed mainly in psychological well-being [20], while in our study differences were also observed in psychological and physical health, especially in vitality and fatigue. Similar to van Weert et al. [38], we also found that fatigue decreased at $\mathrm{T} 1$ for all patients, but especially for patients with inpatient rehabilitation, with moderate effect sizes. In line with Mehnert et al., no improvements were observed for patients in the $\mathrm{A}^{+}$ group [13]. In addition, patients in the $\mathrm{A}^{+}$group even reported a decrease in social functioning and general health. We considered the baseline condition of patients in the IR and those in the $\mathrm{A}^{+}$group to be comparable, as both received medical advice from the hospital's physician. As expected, they had similar medical characteristics and conditions at baseline. Analyses showed that they only differed in age, insurance type, and employment status only in trend. Data from Switzerland indicate that patients with semi-private or private supplementary health insurance are more likely to use inpatient rehabilitation $[8,39,40]$. This might be explained by the higher chance of obtaining rehabilitation more promptly. Patients in the $\mathrm{A}^{-}$group were commonly less impaired and consequently more often employed.

Differences in cancer site should be considered in relation to referral. Patients with cancer of digestive organs, head and neck, and hematological malignancies were more likely to receive referrals during hospital stay, probably due to more severe interventions and, consequently, more impaired participation. Thus, in this subgroup, patients are also more likely to have to wait for rehabilitation or to not receive reimbursement, and thus not receive inpatient rehabilitation at all. In our study, women with breast 
or female genital organ cancers might not have met criteria for referral, as these conditions involve fewer impairments, or their need was not identified. Today, referrals to inpatient rehabilitation for cancer patients are still not based on structured standardized procedures in Switzerland. The implementation of such screening, therefore, is needed to address patients' needs and to render the potential for rehabilitation more reliable [41].

A strength of our study is the longitudinal, naturalistic, cohort design including two different healthcare settings (acute hospital and after acute treatment) and three comparison groups (inpatient rehabilitation and outpatient care with and without advice for rehabilitation). Few studies have combined assessment of acute care and rehabilitation, largely because the transfer of patients to another system increases problems in data collection. In addition, our baseline conditions were comparable between IR and $\mathrm{A}^{+}$in terms of clinical and socio-demographic characteristics. The naturalistic design, used instead of randomization, might lead to heterogenic baseline in some characteristics and scales. However, baseline differences between the groups were controlled by multivariate linear regression analyses. This analysis provides results that are controlled for some of the possible confounders, which approximates equal distribution of them by randomization. Thus, the data converge to those of randomized controlled trials, the gold standard by which to quantify effects [42]. A further strength of our study is that the assessments were standardized, comprehensive, and condition-specific. Finally, the sample sizes of the IR and $\mathrm{A}^{-}$groups were large and the proportion of completed questionnaires was high.

This study has some limitations, which should be considered. The broad inclusion criteria increased the external validity of our results, but such an approach limits the interpretation for specific diagnoses and patient groups. The small sample size of our main control group $\left(\mathrm{A}^{+}\right)$represents a further limitation of this study. Finally, the high rates of dropouts might lead to bias. A particularly high number of dropouts occurred in the IR, mainly related to transfer to hospital and severe decrease of health status.

\section{Conclusion}

These findings support recommendations for inpatient cancer rehabilitation as an effective management after initial acute treatment for those patients whose health is too severely affected for discharge directly at home. Structured standardized assessments might be required for the identification of patients' needs and the potential for rehabilitation, and such assessments should be implemented on a regular basis.

\section{Acknowledgement}

We thank all patients in this study for completing the sets of questionnaires. We are grateful to the participating departments of the University Hospital Zurich (Department of Cranio-Maxillo-facial and Oral Surgery, Department of Gynaecology, Department of Oncology, Department of Otorhinolaryngology and Head and Neck Surgery, Department of Radiation of Oncology, Department of Thoracic Surgery, Department of Visceral and Transplant Surgery), the Balgrist Hospital Zurich, the Oncology Center Hirslanden Zurich, the Susenberg Klinik, the Zürcher RehaZentrum Davos, and the RehaClinic Bad Zurzach.

\section{Funding}

This study was financially supported by the Zurzach Rehabilitation Foundation SPA, Bad Zurzach and by the University of Zurich, Switzerland.

\section{Competing interests}

All authors declare no conflict of interest.

\section{References}

1. Van Weert E, Hoekstra-Weebers J, Grol B, Otter R, Arendzen HJ, Postema K, et al. A multidimensional cancer rehabilitation program for cancer survivors: Effectiveness on health-related quality of life. J Psychosom Res. 2005; 58:485-96.

2. Carr D, Goudas L, Lawrence D, Pirl W, Lau J, DeVine D, et al. Management of cancer symptoms: pain, depression, and fatigue. Evid Rep Technol Assess (Summ). 2002:1-5.

3. Cromes GF. Implementation of interdisciplinary rehabilitation. Rehab Couns Bull. 1978; 21:230-7.

4. Cheville AL. Cancer rehabilitation. Semin Oncol. 2005; 32:219-24.

5. World Health Organization. Disability prevention and rehabilitation: Report of the WHO expert committee on disability prevention and rehabilitation. Tech Rep Ser. 1981; 668.

6. Khan F, Amatya B, Ng L, Drummond K, Olver J. Multidisciplinary rehabilitation after primary brain tumour treatment. Cochrane Database Syst Rev 2013;1:CD009509. doi: 10.1002/14651858.CD009509.pub2

7. Mewes JC, Steuten LMG, Ijzerman MJ, van Harten WH. Effectiveness of multidimensional cancer survivor rehabilitation and cost-effectiveness of cancer rehabilitation in general: a systematic review. Oncologist. 2012; 17:1581-93.

8. [Internet] Klinik Susenberg. Zahlen n.d. http://www.susenbergklinik.ch/ ueber-uns/zahlen/

9. [Internet] Spitalplanung Rehabilitation 2014 Versorgungsbericht n.d. http://www.sg.ch/home/gesundheit/gesundheitsversorgung/spitalliste/j cr_content/Par/downloadlist/DownloadListPar/download.ocFile/Versorgu ngsbericht_Rehabilitation.pdf

10. [Internet] oncoreha.ch n.d. http://www.oncoreha.ch/about/

11. Speck RM, Courneya KS, Masse LC, Duval S, Schmitz KH. An update of controlled physical activity trials in cancer survivors: a systematic review and meta-analysis. J Cancer Surviv. 2010; 4:87-100.

12. Spence RR, Heesch KC, Brown WJ. Exercise and cancer rehabilitation: a systematic review. Cancer Treat Rev. 2010; 36:185-94.

13. Mehnert A, Veers S, Howaldt D, Braumann K-M, Koch U, Schulz K-H. Effects of a physical exercise rehabilitation group program on anxiety, depression, body image, and health-related quality of life among breast cancer patients. Onkologie. 2011; 34:248-53.

14. Huang ME, Sliwa JA. Inpatient rehabilitation of patients with cancer: efficacy and treatment considerations. PM\&R. 2011; 3:746-57.

15. Teichmann JV. Oncological rehabilitation: evaluation of the efficiency of inpatient rehabilitation. Rehabil. 2002; 41:53-63.

16. Riesenberg H, Lübbe AS. In-patient rehabilitation of lung cancer patients--a prospective study. Support Care Cancer. 2010; 18:877-82. 
17. McEwen SE, Elmi S, Waldman M, Bishev M. Inpatient oncology rehabilitation in Toronto: A descriptive 18-month retrospective record review. Support Care Cancer. 2012; 20:1541-7.

18. Baili P, Hoekstra-Weebers J, Van Hoof E, Bartsch HH, Travado L, Garami M, et al. Cancer rehabilitation indicators for Europe. Eur J Cancer. 2013; 49:1356-64.

19. Strauss-Blasche G, Gnad E, Ekmekcioglu C, Hladschik B, Marktl W. Combined inpatient rehabilitation and spa therapy for breast cancer patients: effects on quality of life and CA 15-3. Cancer Nurs. 2005; 28:390-8.

20. Weis J, Moser MT BH. Goal-orientated evaluation of inpatient rehabilitation programs for women with breast cancer (ZESOR-study). In: Jäckel WH, Bengel J, Herdt J E, editor. Res. Rehabil. Results from a Res. Netw. southwest Ger., Stuttgart: Schattauer; 2006.

21. Paul K, Buschbacher R. Cancer rehabilitation: increasing awareness and removing barriers. Am J Phys Med Rehabil. 2011; 90:S1-4.

22. Cole RP, Scialla SJ, Bednarz L. Functional recovery in cancer rehabilitation. Arch Phys Med Rehabil. 2000; 81:623-7.

23. [Internet] Fachtagung «Onkologische Rehabilitation - Versorgungsmodelle in der Schweiz» n.d. http://www.krebsliga.ch/de/uber_uns/ medienschaffende $2 /$ medienmitteilungen.cfm?uNewsID $=584$

24. Ture M, Barth J, Angst F, Aeschlimann A, Schnyder U, Zerkiebel N, et al. Use of inpatient rehabilitation for cancer patients in Switzerland: Who undergoes cancer rehabilitation? Swiss Med Wkly. 2015; 4:145:w14214.

25. Paul K, Buschbacher R. Cancer rehabilitation: increasing awareness and removing barriers. Am J Phys Med Rehabil. 2011; 90:S1-4.

26. Kessler J, Markowitsch HJ, Denzler P. Mini-Mental-Status-Test (MMST). Göttingen: Beltz Test GmbH; 2000.

27. Sobin LH, Gospodarowicz M, Wittekind C. TNM classification of malignant tumours. $7^{\text {th }}$ ed. Oxford: Wiley-Blackwell; 2009.

28. Bullinger M, Kirchberger I. SF-36 Fragebogen zum Gesundheitszustand. Handanweisung. Göttingen: Hogrefe; 1998.

29. Ware JE, Snow KK, Kosinski M, Gandek B. SF-36 Health survery: manual and interpretation guide. In QualityMetric Incorporated. $3^{\text {rd }}$ ed. USA: Lincoln RI; 2013.

30. Yellen SB, Cella DF, Webster K, Blendowski C, Kaplan E. Measuring fatigue and other anemia-related symptoms with the Functional Assessment of Cancer Therapy (FACT) measurement system. J Pain Symptom Manage. 1997; 13:63-74.

31. Snaith RP. The Hospital Anxiety And Depression Scale. Heal Qual Life Outcomes. 2003;1:29.

32. Hinz A, Brähler E. Normative values for the hospital anxiety and depression scale (HADS) in the general german population. J Psychosom Res. 2011; 71:74-8.

33. Borenstein M. Effect sizes for continuous data. In: Cooper H, Hedges L V, Valentine JC, editors. Handb. Res. Synth. meta-analysis. 2nd ed., New York: Russell Sage Foundation; 2009.

34. Cohen J. Statistical power analysis for the behavioral sciences. $2^{\text {nd }}$ ed. New York: Lawrence Erlbaum Associates; 1988.

35. Haaf HG. Findings on the Effectiveness of Rehabilitation. Rehabil. 2005; 44:e1-20.

36. Heim ME, v d Malsburg ML, Niklas A. Randomized controlled trial of a structured training program in breast cancer patients with tumor-related chronic fatigue. Onkologie. 2007; 30:429-34.

37. Hartmann U, Muche R, Reuss-Borst M. Effects of a step-by-step inpatient rehabilitation programme on quality of life in breast cancer patients. A prospective randomised study. Onkologie. 2007; 30:177-82.

38. van Weert E, May AM, Korstjens I, Post WJ, van der Schans $\mathrm{CP}$, van der Borne $\mathrm{B}$, et al. Cancer-related fatigue and rehabilitation: a randomized controlled multicenter trial comparing physical training combined with cognitive-behavioral therapy with physical training only and with no Intervention. Phys Ther. 2010; 90:1413-25.

39. [Internet] Klinik Schloss Mammern. Zahlen \& Fakten n.d. http://www.klinik-schloss-mammern.ch/de/zahlen fakten.61

40. [Internet] Reha Chrischona. Jahresbericht Reha Chrischona 2013 n.d. http://www.reha.buespi.ch/files/G8KZCEI/Jahresbericht-Reha-Chrischona2013

41. Carlson LE, Waller A, Mitchell AJ. Screening for distress and unmet needs in patients with cancer: Review and Recommendations. J Clin Oncol. 2012; 30:1160-77.

42. Borenstein M, Hedges L, Higgins J, Rothstein HR. Computing effect sizes for meta-analysis. Chichester: John Wiley \& Sons, Ltd. 2009. 